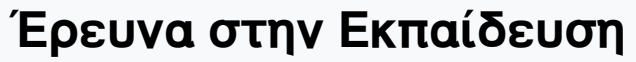

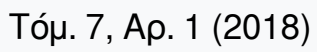

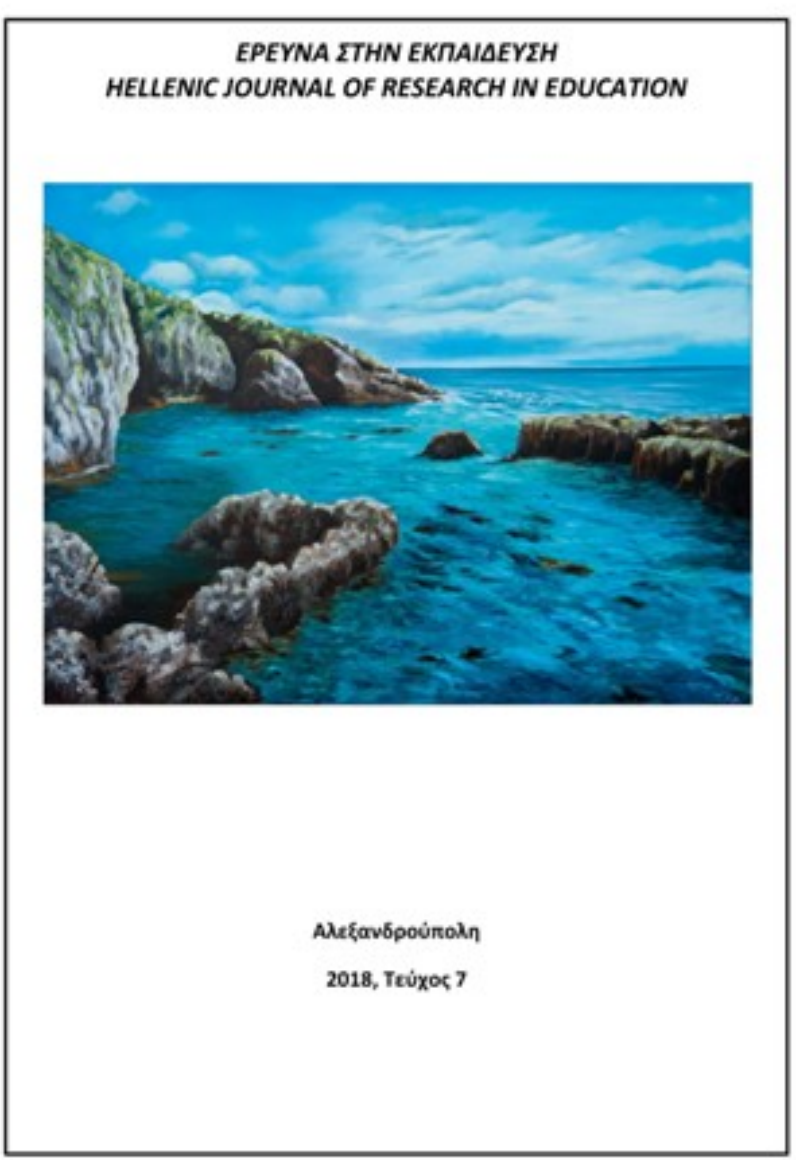

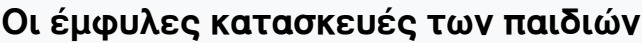

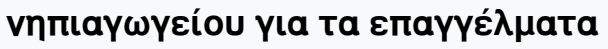

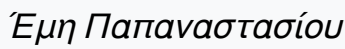

doi: $10.12681 /$ hjre.14232

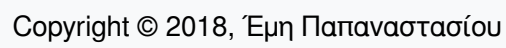

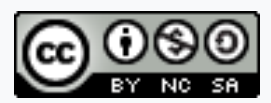

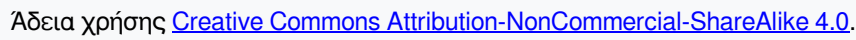

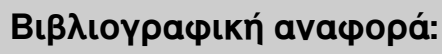

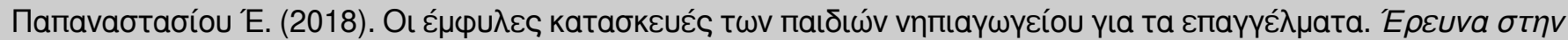

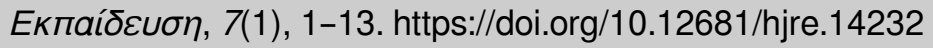




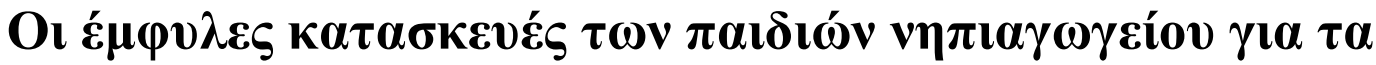 $\varepsilon \pi \alpha \gamma \gamma \tilde{\varepsilon} \lambda \mu \alpha \tau \alpha$
}

\author{
$\Pi \alpha \pi \alpha v \alpha \sigma \tau \alpha \sigma i ́ o v ' E \mu \eta$

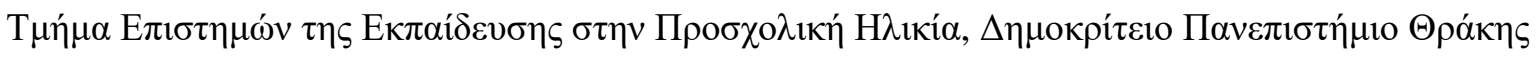

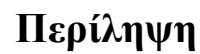

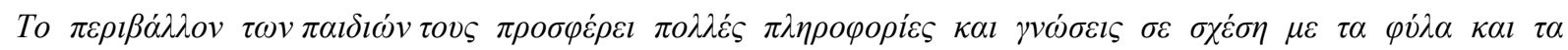

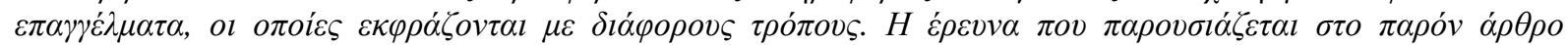

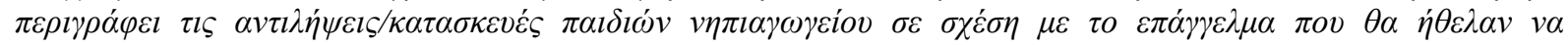

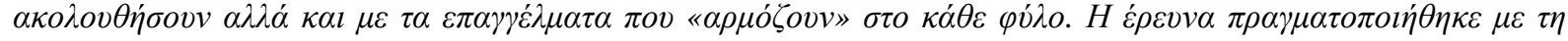

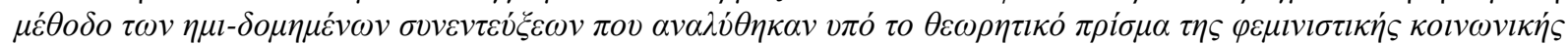

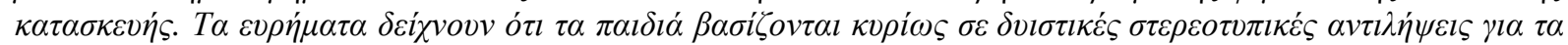
$\varphi v ́ \lambda \alpha \kappa \alpha l \tau \eta v \varepsilon \pi \imath \lambda o \gamma \dot{\prime} \varepsilon \pi \alpha \gamma \gamma \varepsilon \dot{\lambda} \mu \alpha \tau o \varsigma$.

\begin{abstract}
Children get information and knowledge from their cultural environment about gender and work, that are expressed in various ways. The research presented in this paper describes children's constructions about the profession they wish to do in the future and the professions that are more «suitable» to each gender. Feminist social constructionism theory was used to analyse semi-stuctured interviews with children. The findings suggest that children's constructions are mainly based on dualist stereotypical perceptions about genders and professional choice.
\end{abstract}

(C) 2018, E. П $\alpha \pi \alpha v \alpha \sigma \tau \alpha \sigma i ́ o v$

A $\delta \varepsilon 1 \alpha$ CC-BY-SA 4.0

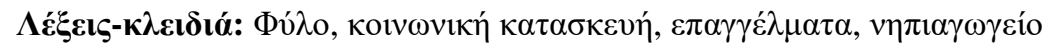

Key words: Gender, social constructionism, professions, pre-school

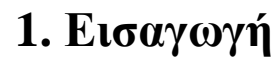

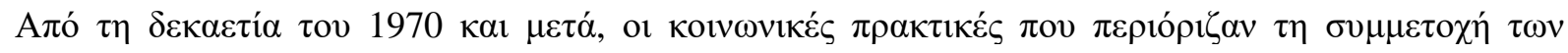

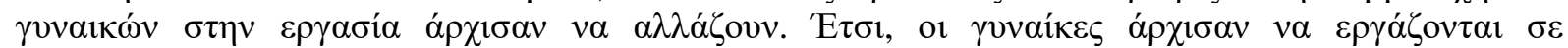

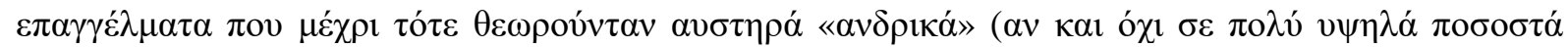

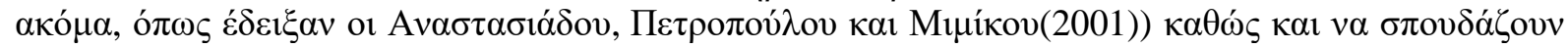

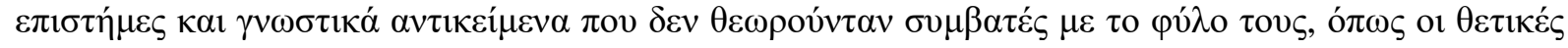

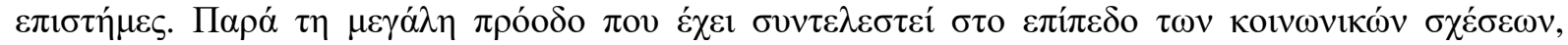

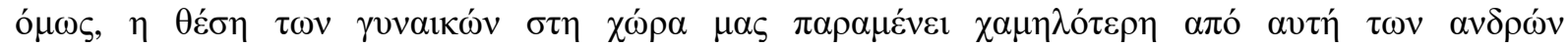

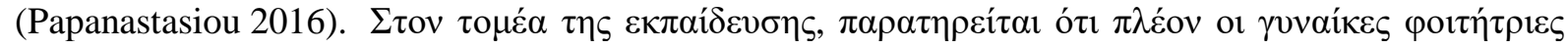

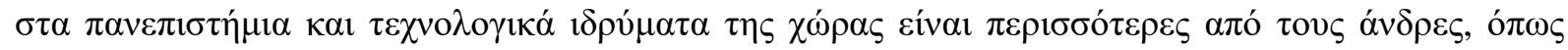

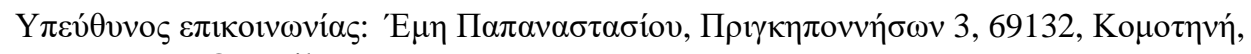
emmypapapn@gmail.com

URL: http://ejournals.epublishing.ekt.gr/index.php/hjre/index 


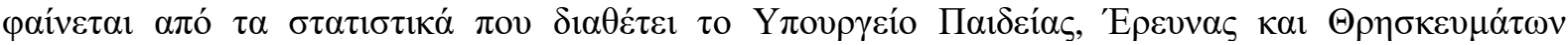

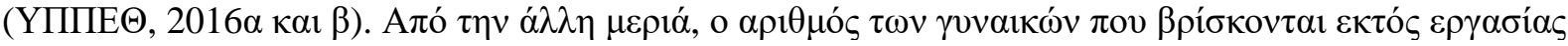

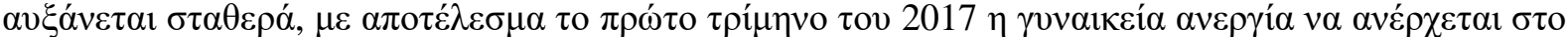

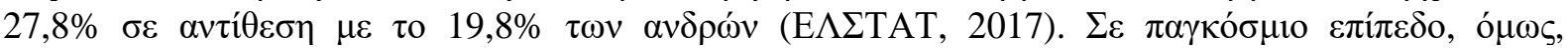

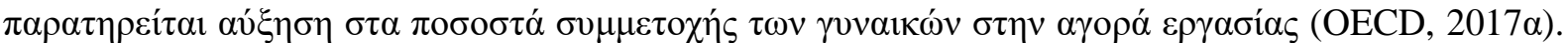

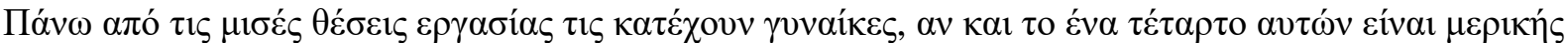

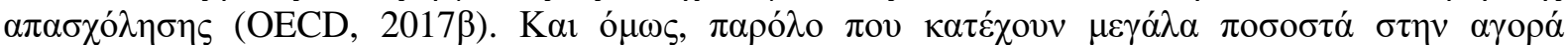

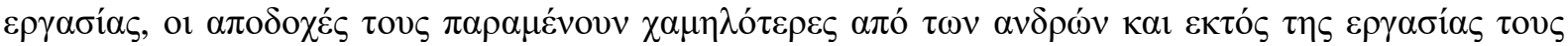

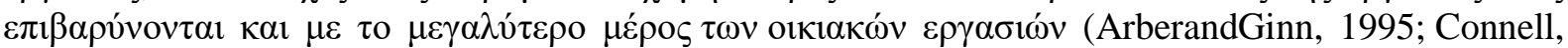

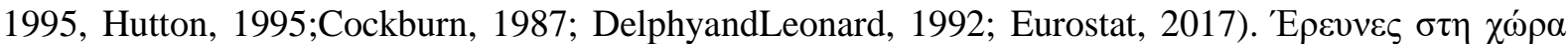

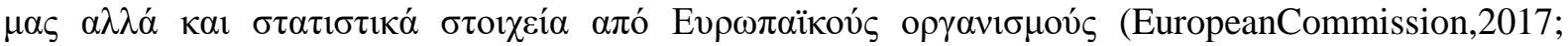

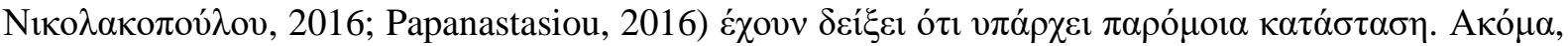

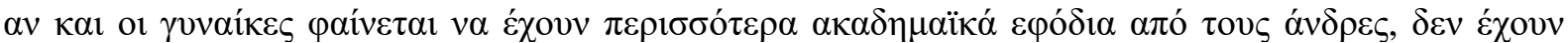

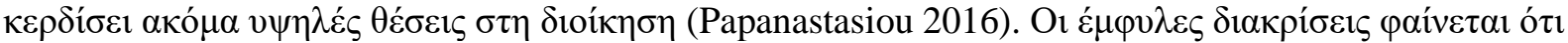

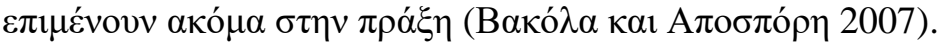

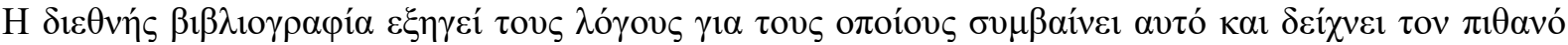

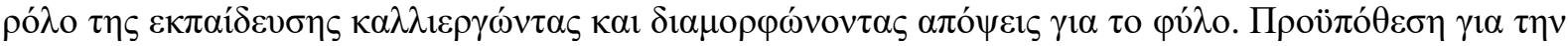

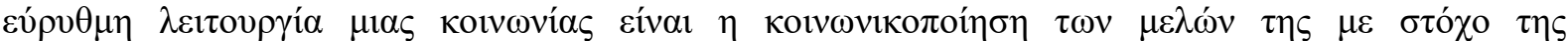

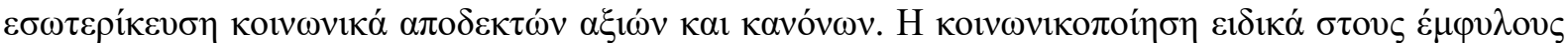

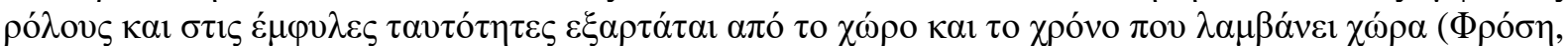

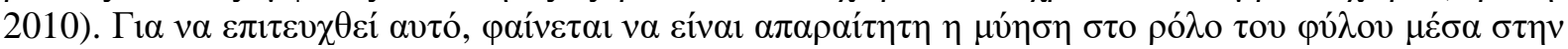

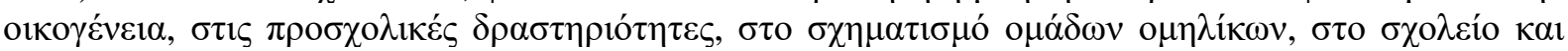

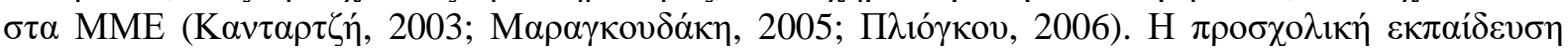

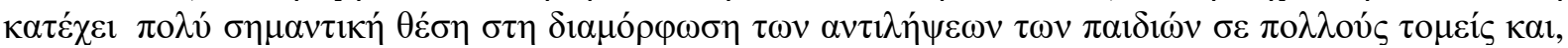

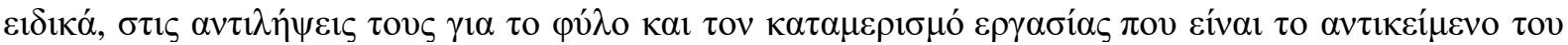

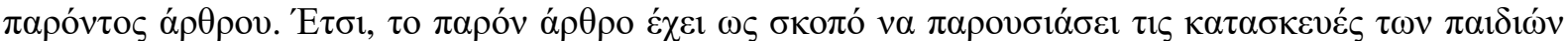

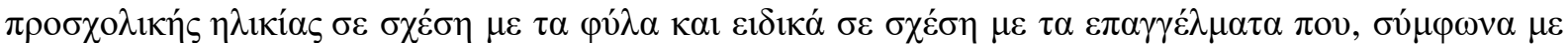

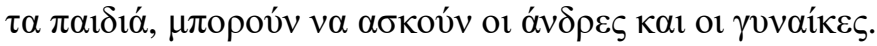

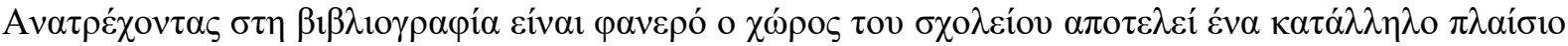

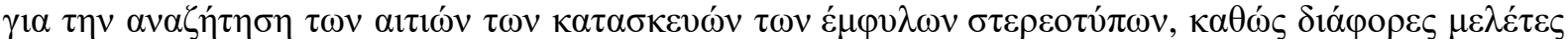

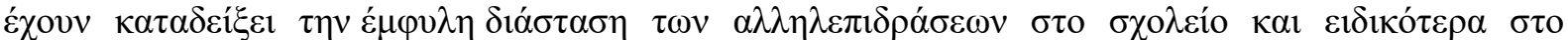

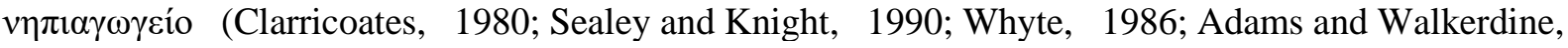

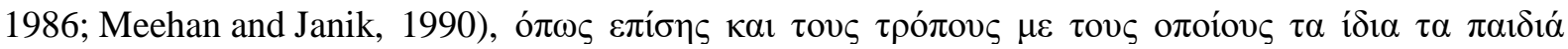

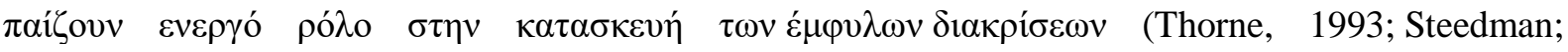

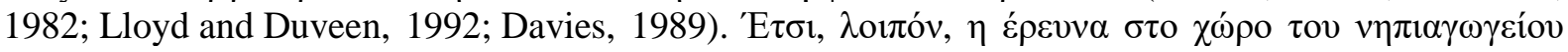

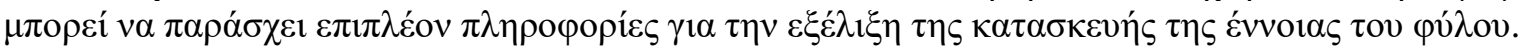

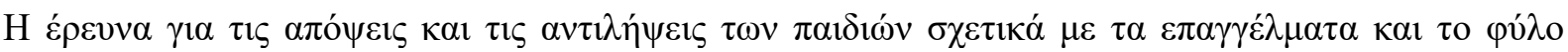

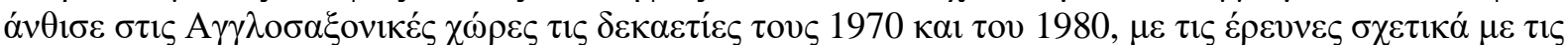

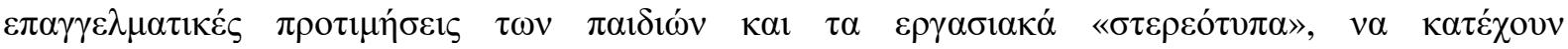

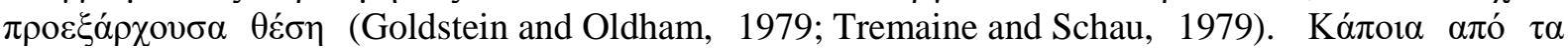

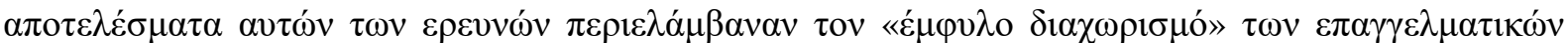

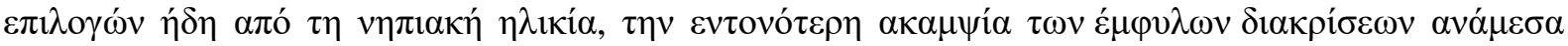

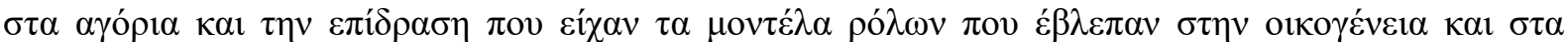

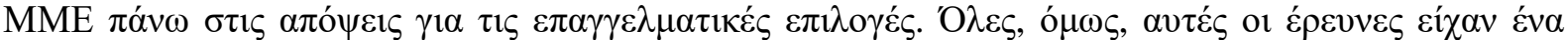

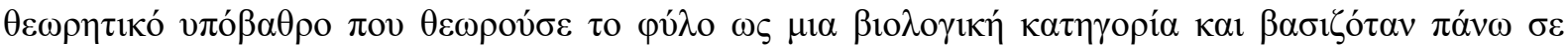

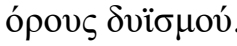

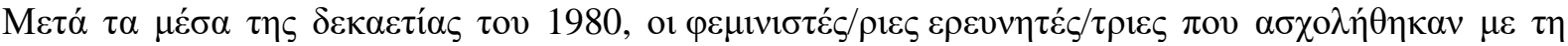

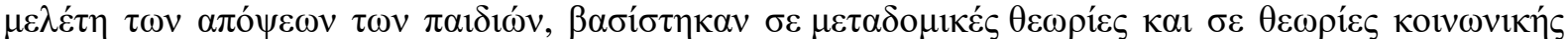

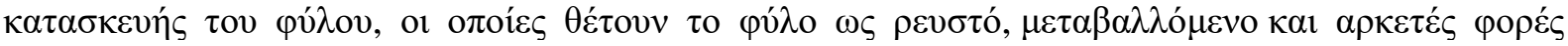

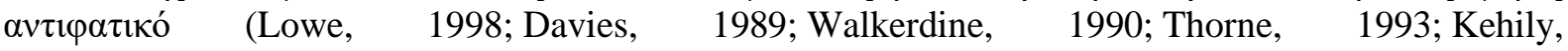




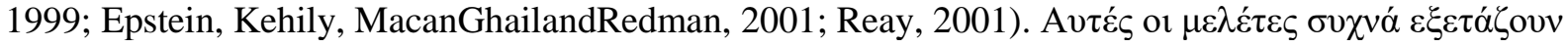

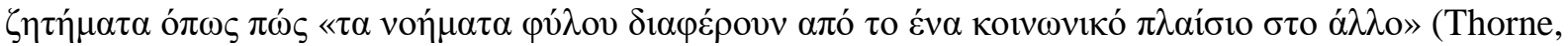

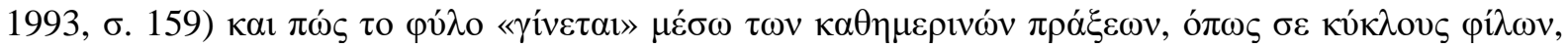

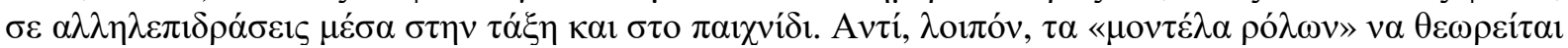

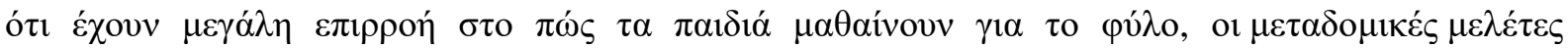

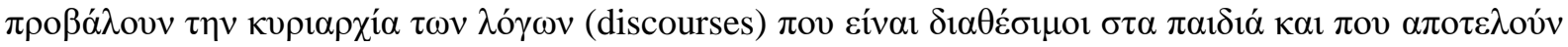

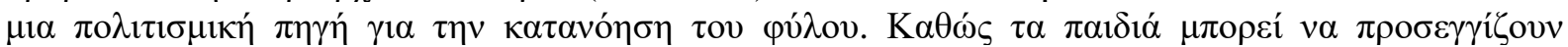

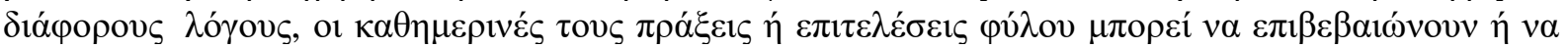

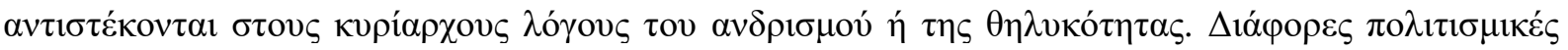

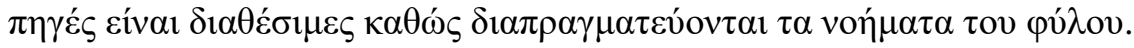

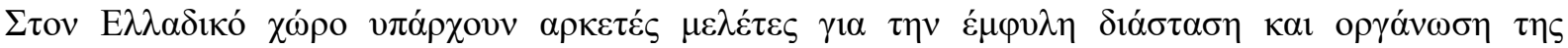

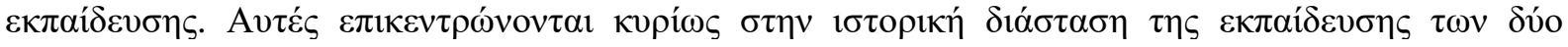

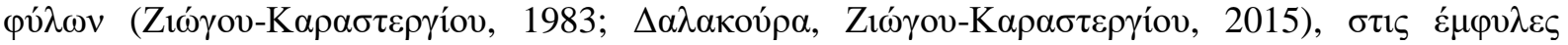

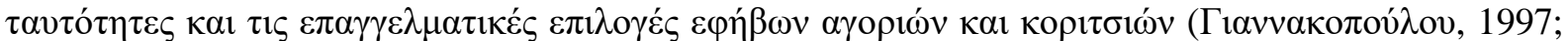

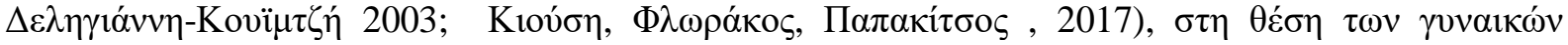

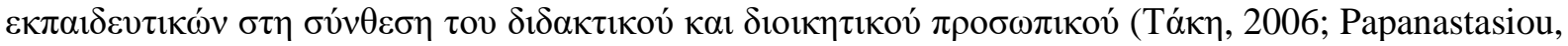

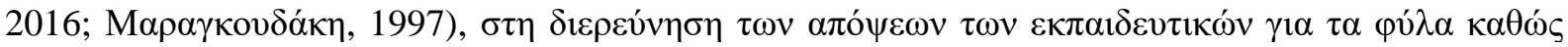

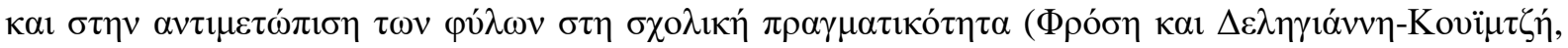

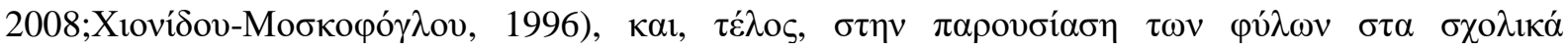

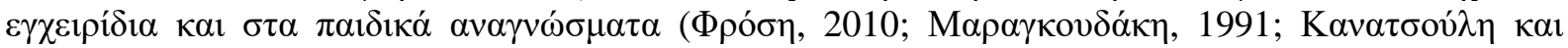

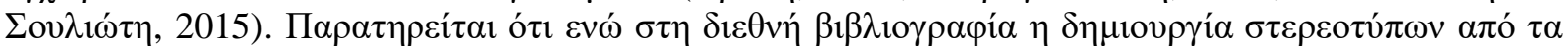

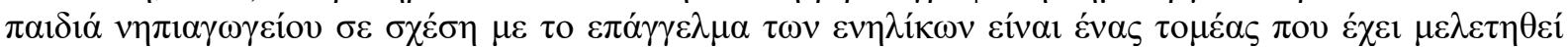

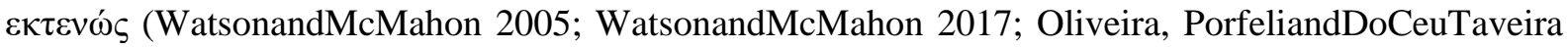

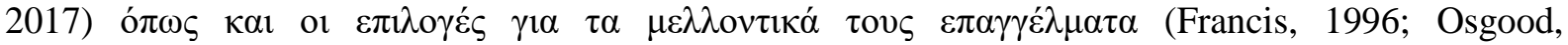

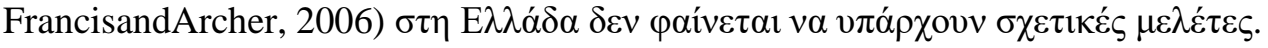

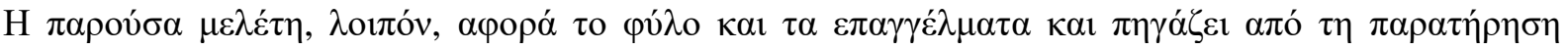

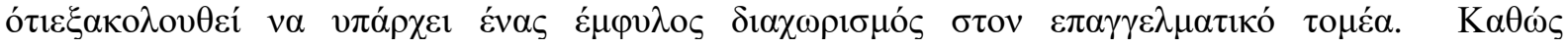

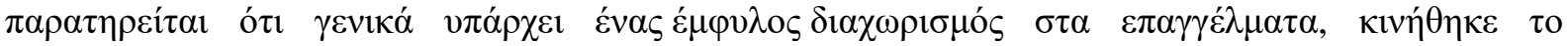

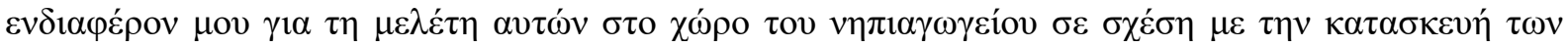

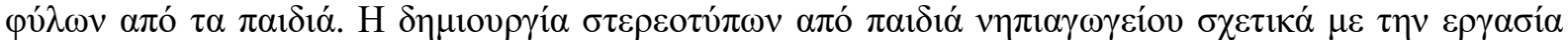

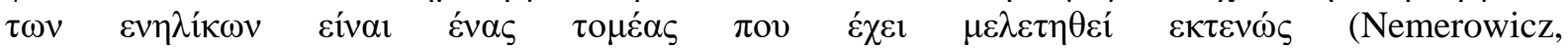
1979; Adams and Walkerdine, 1986; Rosenthal and Chapman, 1982; Tremaine, 1982), ó $\pi \omega \varsigma$ каl or

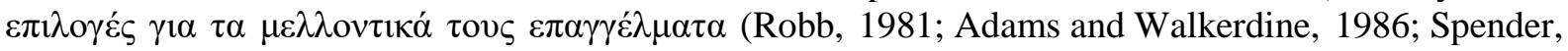

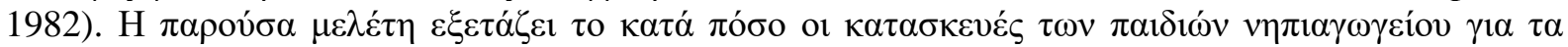

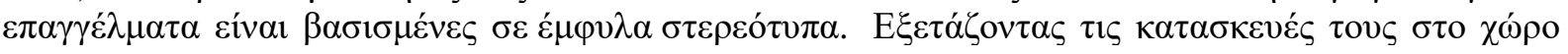

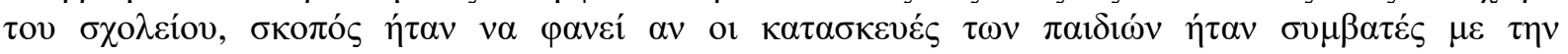

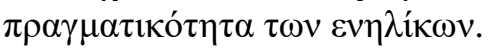

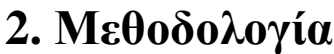

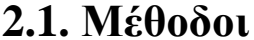

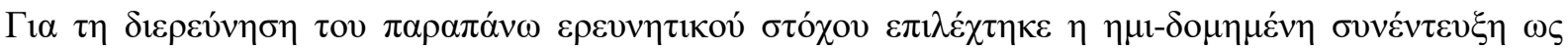

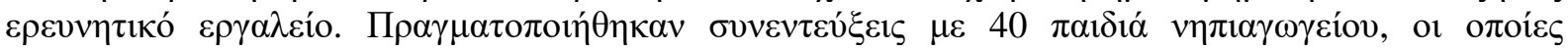

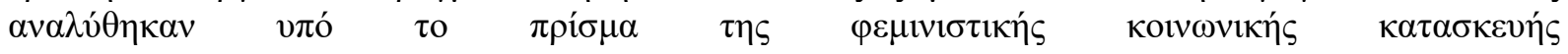

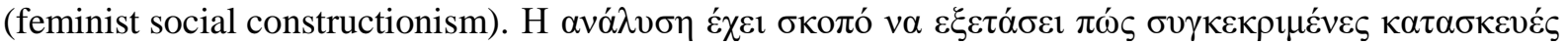

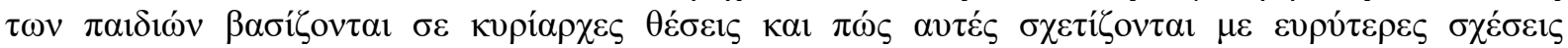

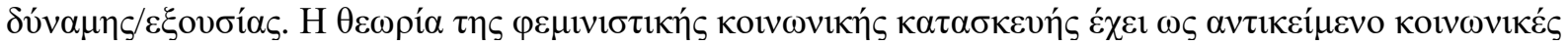

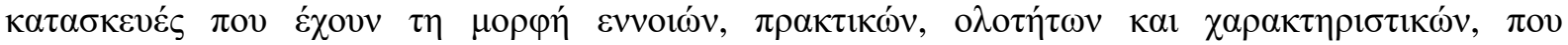

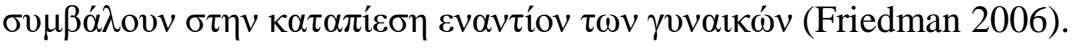




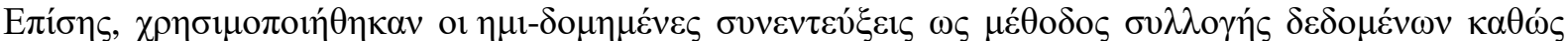

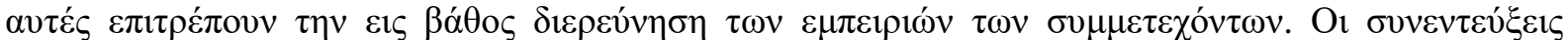

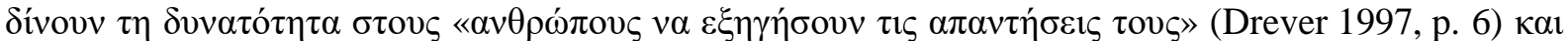

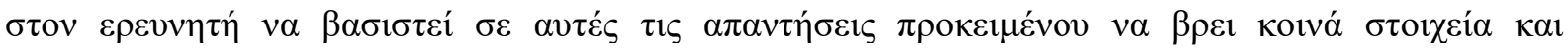

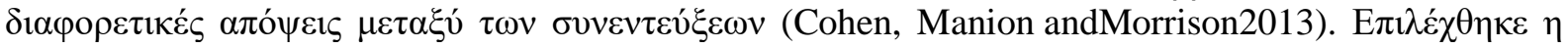

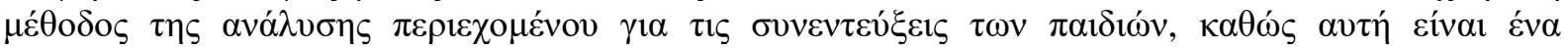

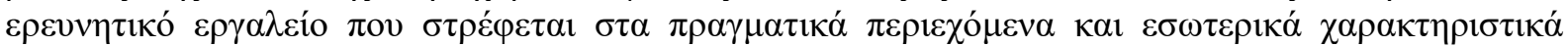

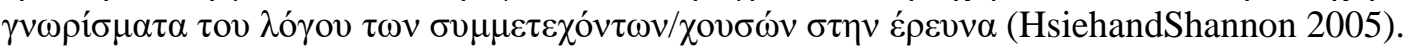

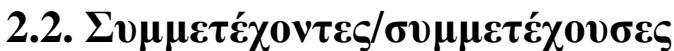

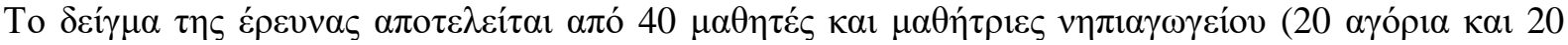

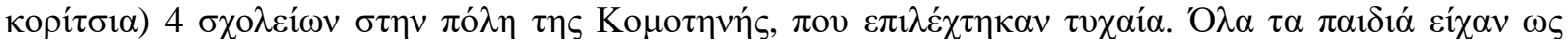

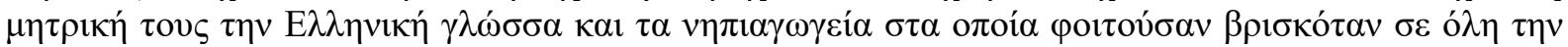

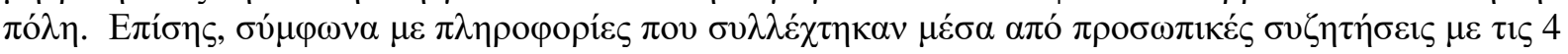

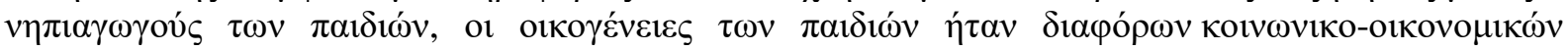

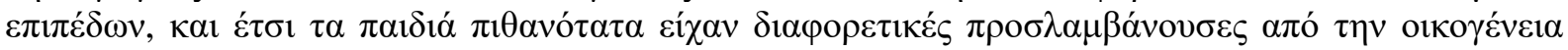

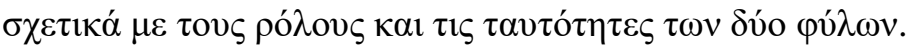

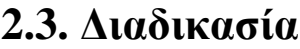

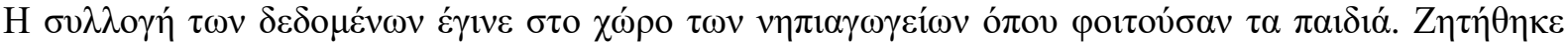

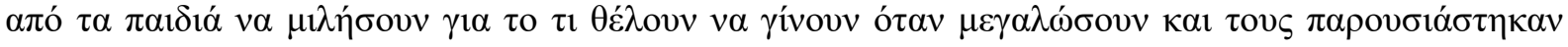

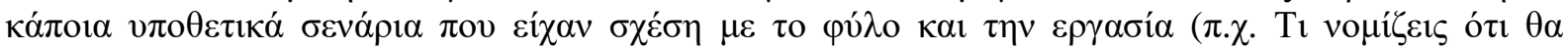
$\gamma 1 v o ́ \tau \alpha \nu \alpha v$ ol

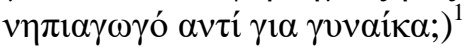

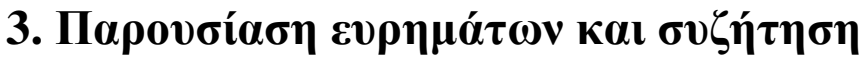

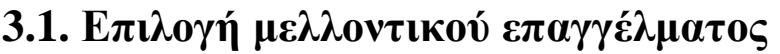

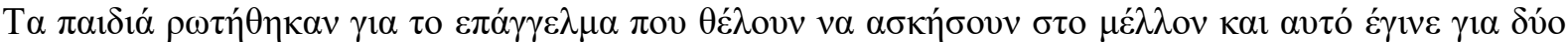

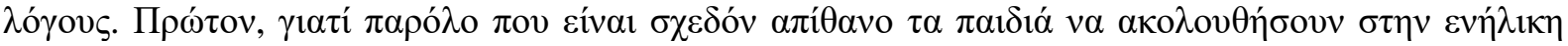

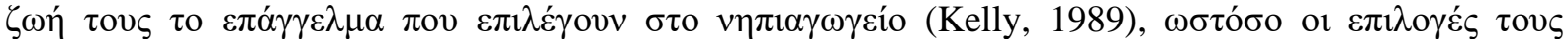

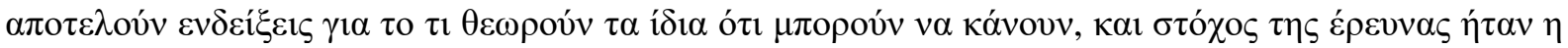

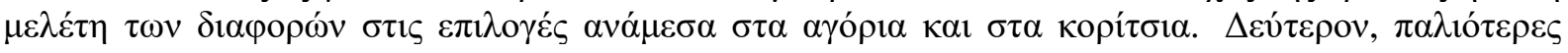

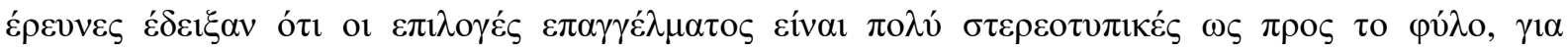

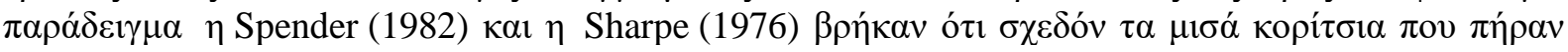

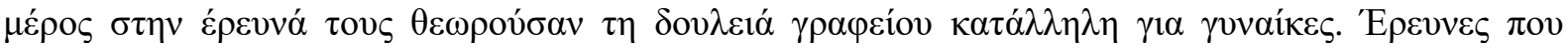

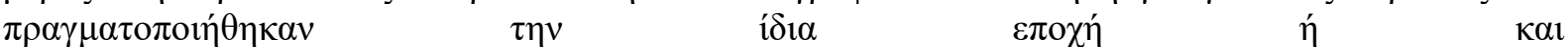

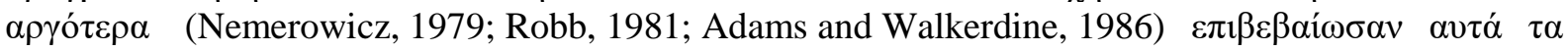

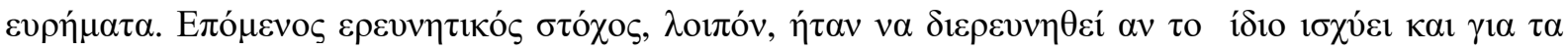

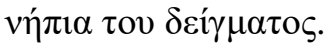

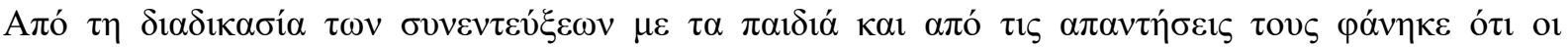

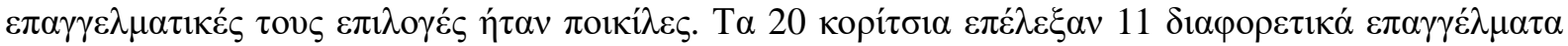

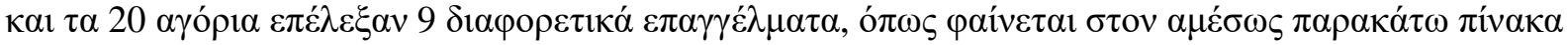
1.

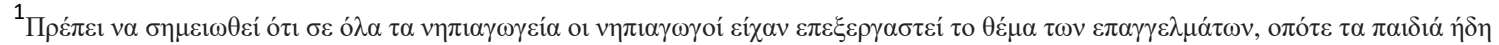

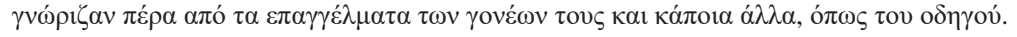




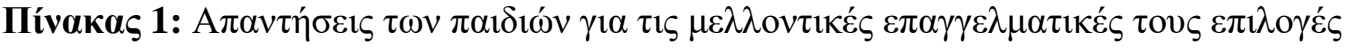

\begin{tabular}{|c|c|}
\hline 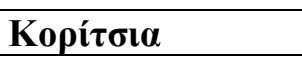 & 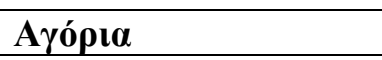 \\
\hline 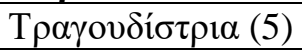 & 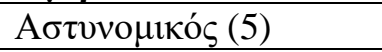 \\
\hline$\Delta \alpha \sigma \kappa \alpha ́ \lambda \alpha(3)$ & 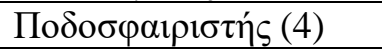 \\
\hline 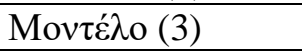 & 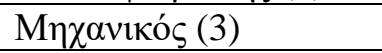 \\
\hline Kо $\mu \mu \omega ́ \tau \rho ı \alpha(2)$ & 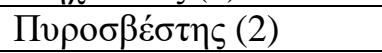 \\
\hline 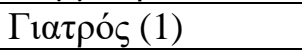 & 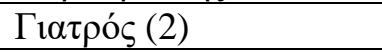 \\
\hline 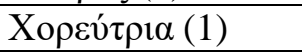 & 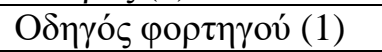 \\
\hline 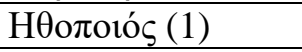 & 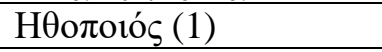 \\
\hline 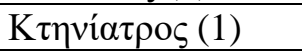 & 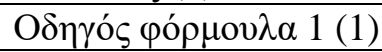 \\
\hline 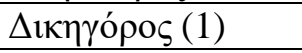 & 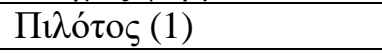 \\
\hline \multicolumn{2}{|l|}{ 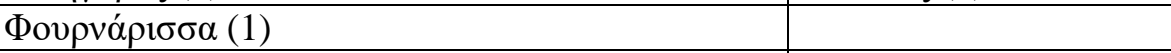 } \\
\hline$\Pi \omega \lambda \eta \dot{\tau} \tau \iota \alpha(1)$ & \\
\hline
\end{tabular}

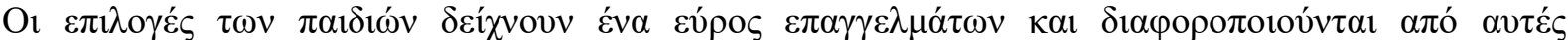

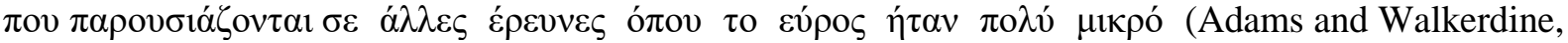

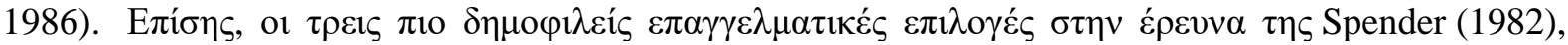

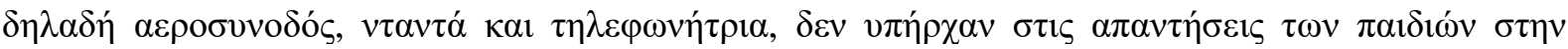

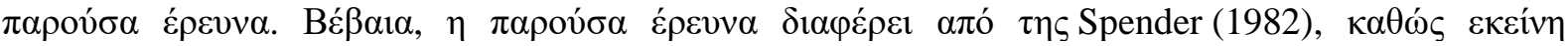

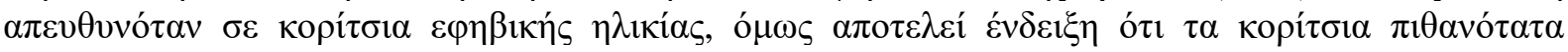

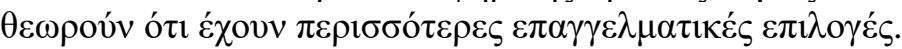

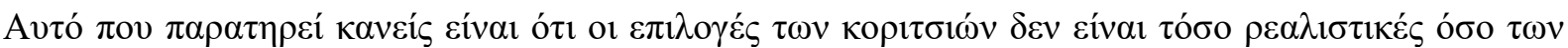

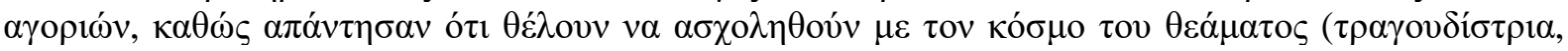

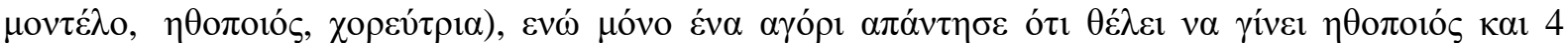

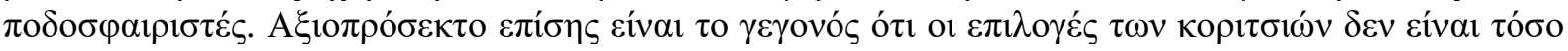

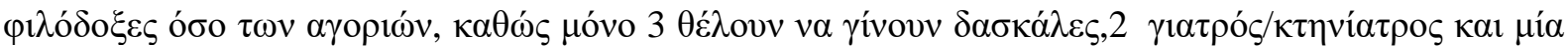

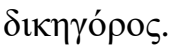

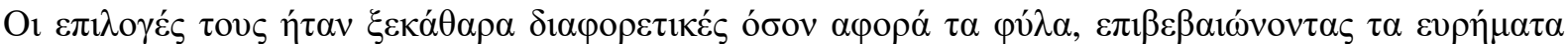

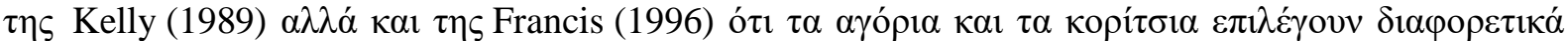

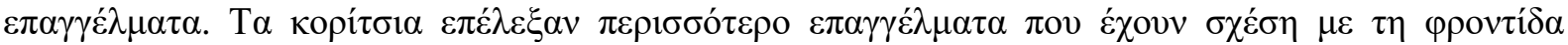

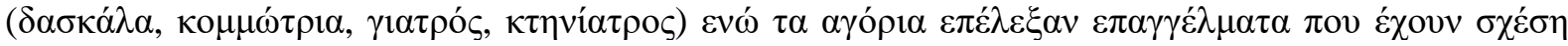

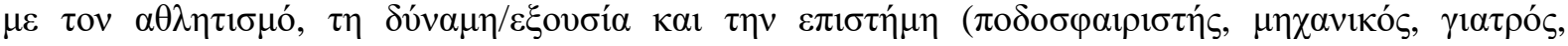

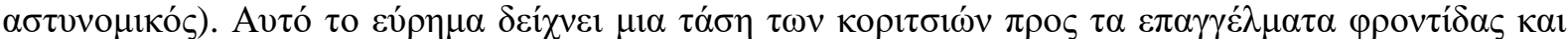

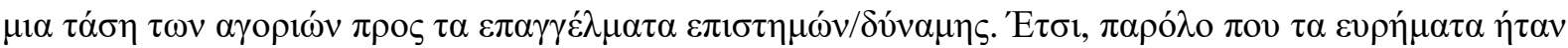

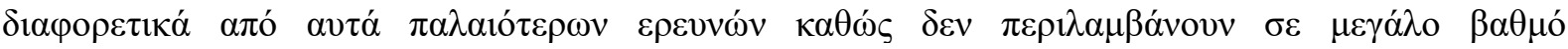

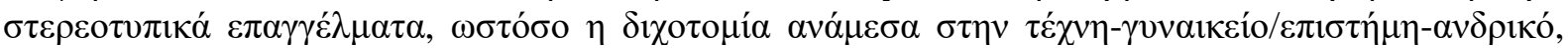

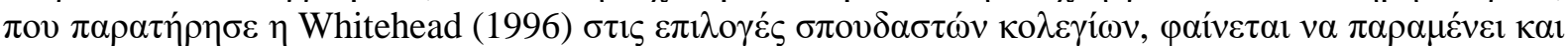

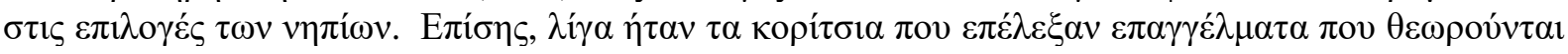

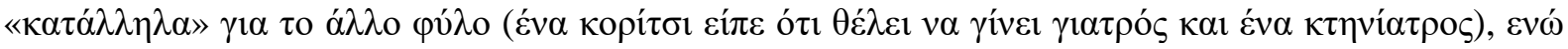

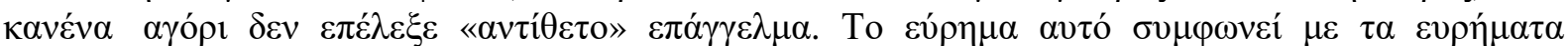

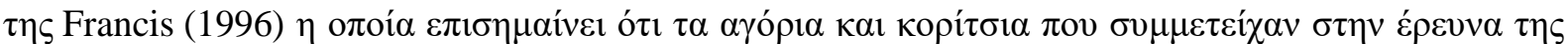

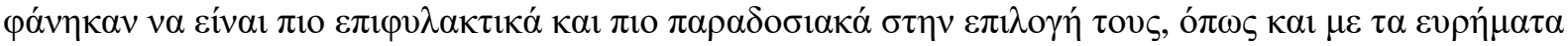

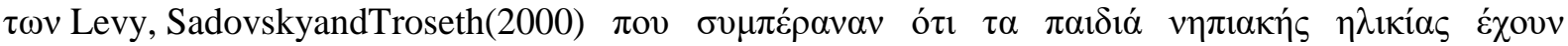

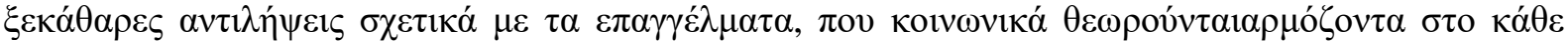
pú̉o. 


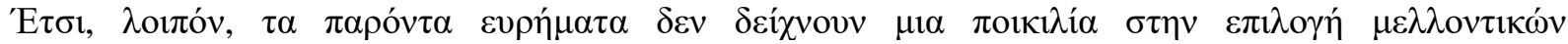

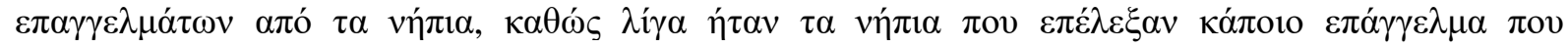

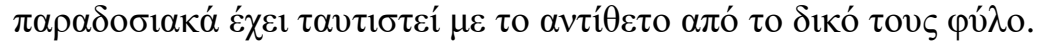

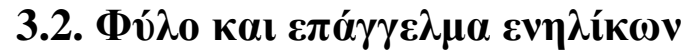

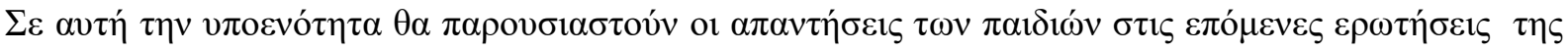

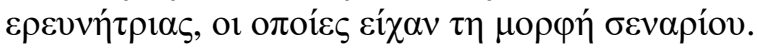

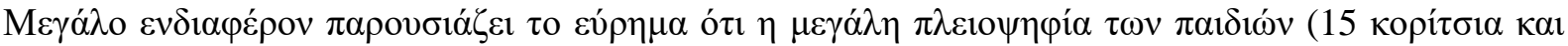

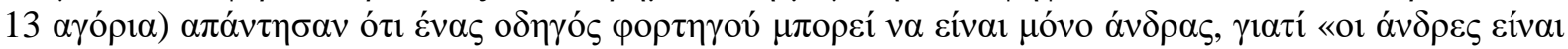

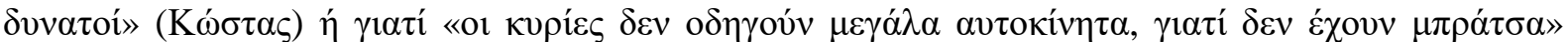

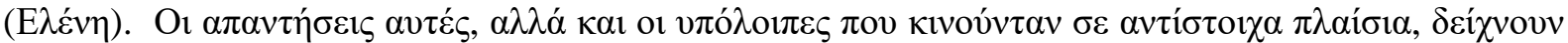

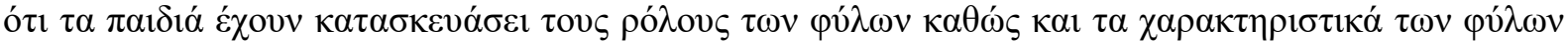

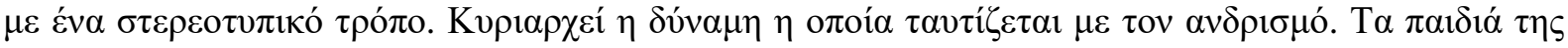

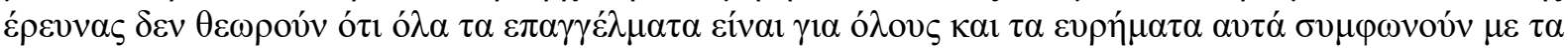

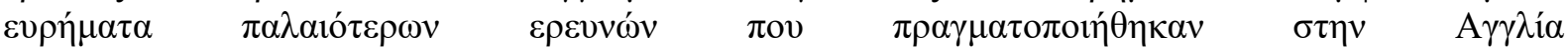

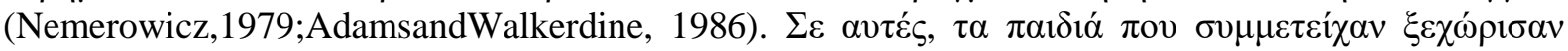

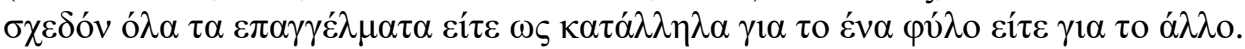

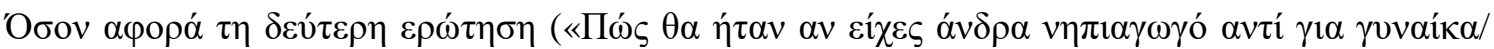

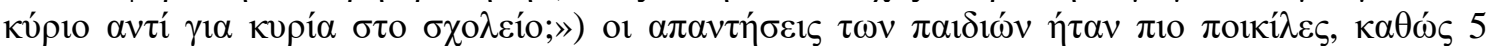

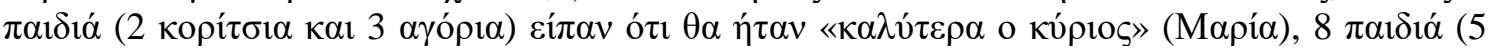

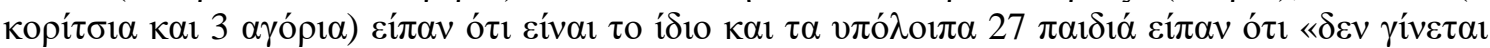

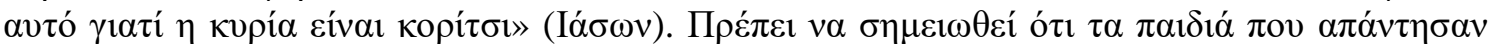

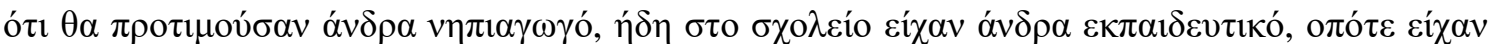

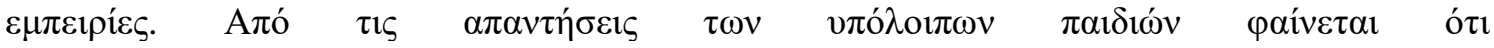

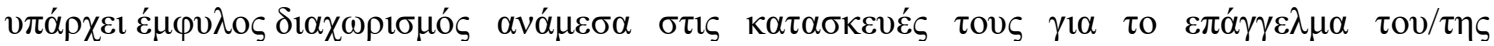

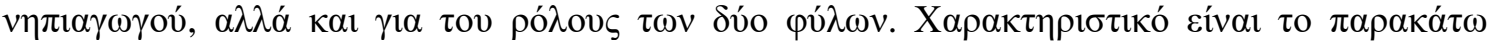

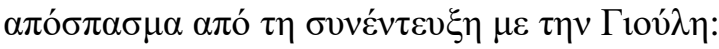

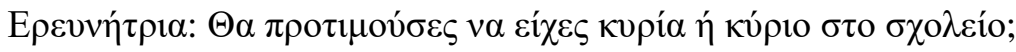

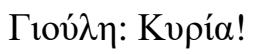

E: Гıน⿰彳́;

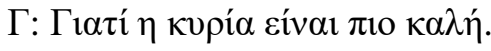

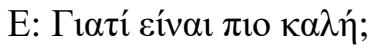

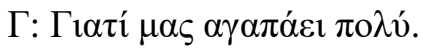

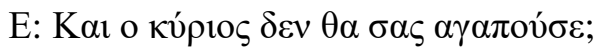

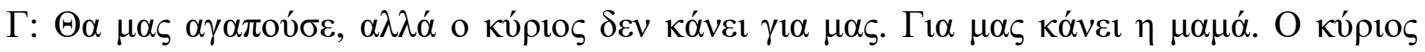

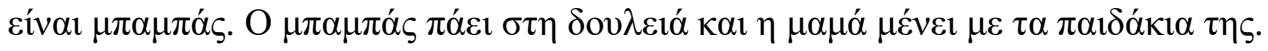

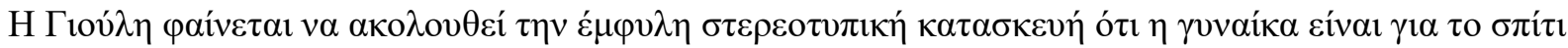

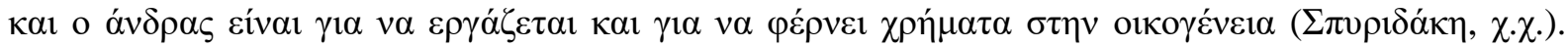

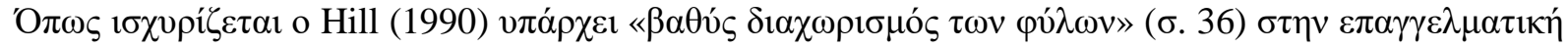
$\zeta \omega \eta ́$. 


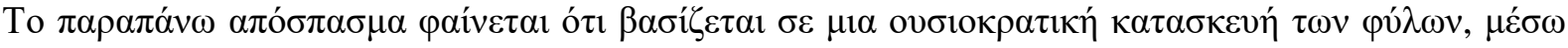

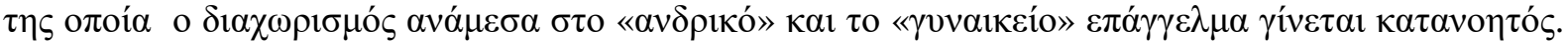

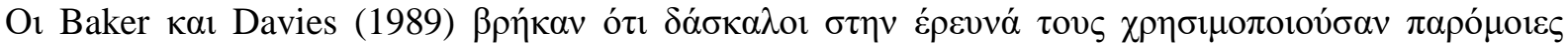

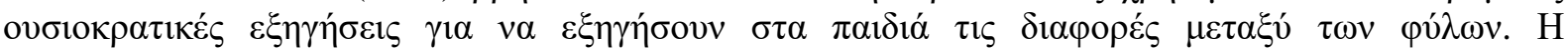

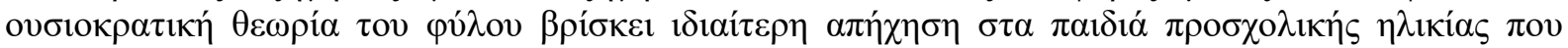

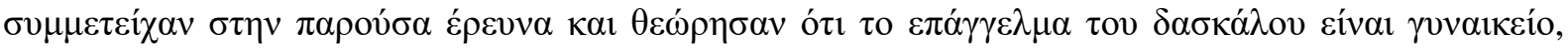

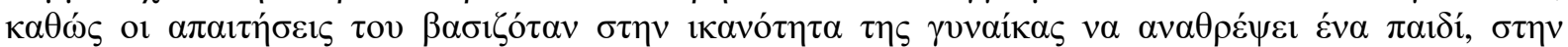

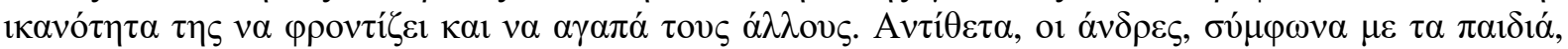

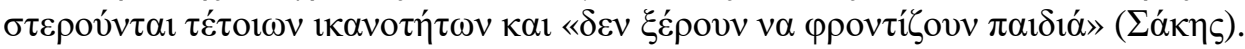

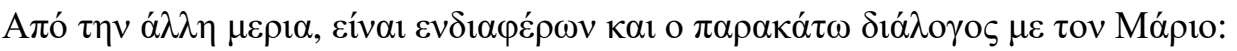

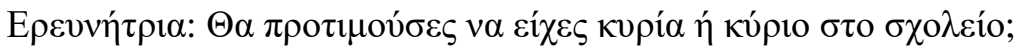

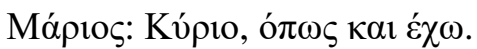

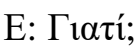

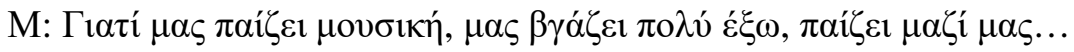

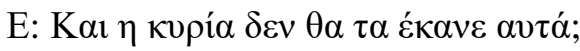

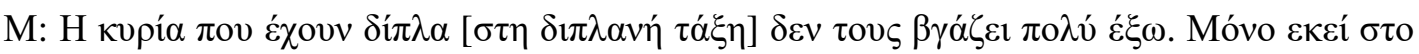
$\theta \dot{\varepsilon} \alpha \tau \rho \circ \pi \mathrm{ov} \pi \dot{\eta} \gamma \alpha \mu \varepsilon$.

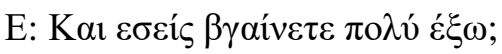

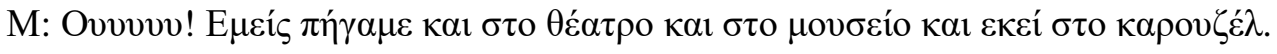

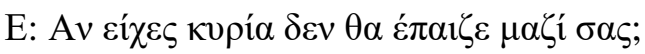

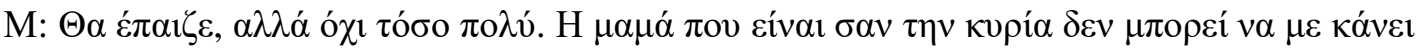

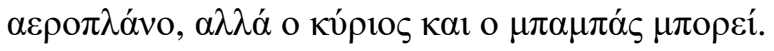

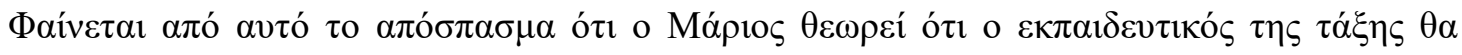

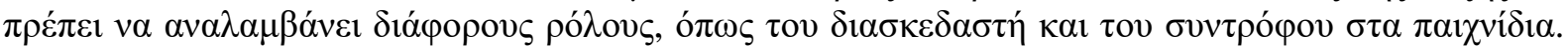

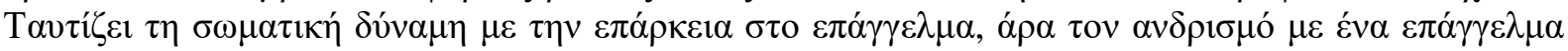

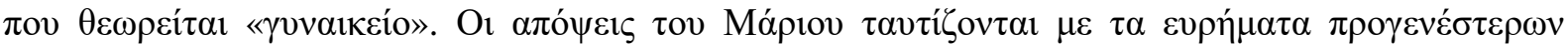

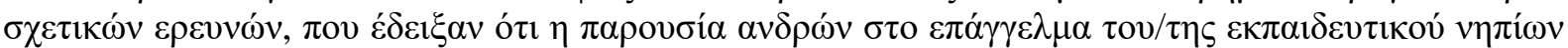

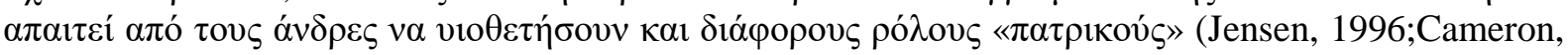
2001).

\section{4. Елíl}

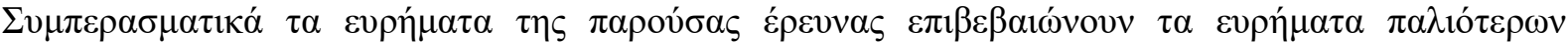

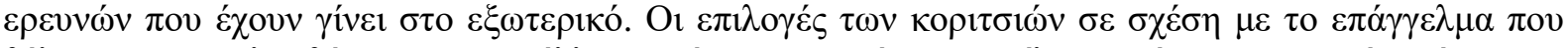

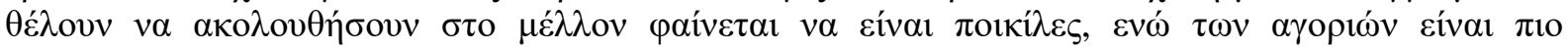

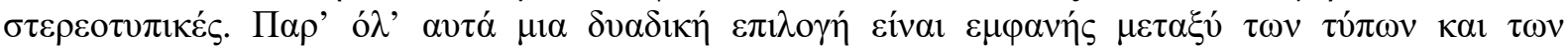

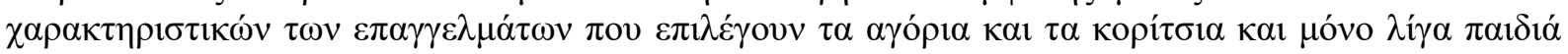

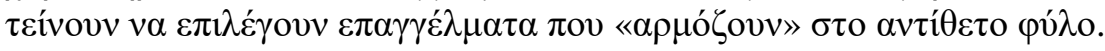




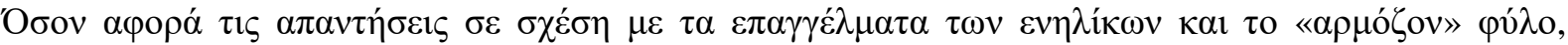

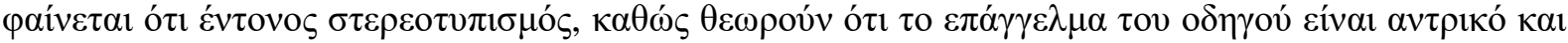

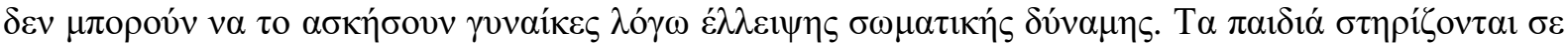

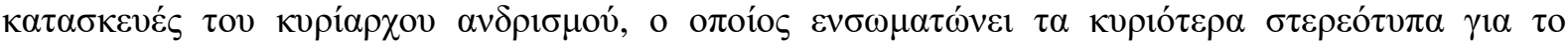

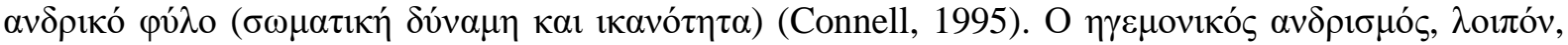

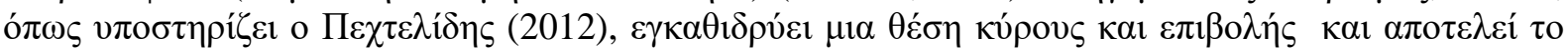

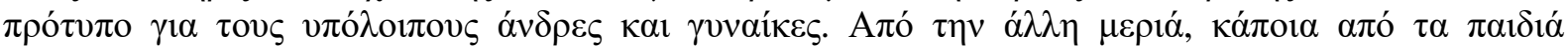

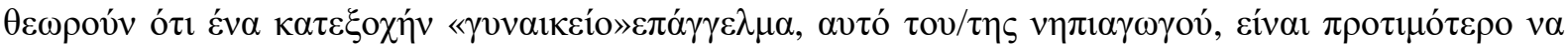

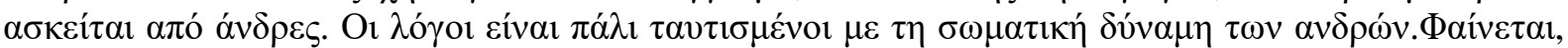

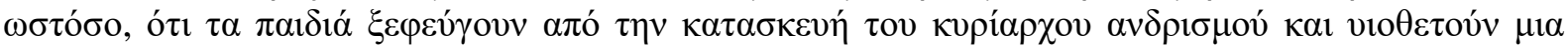

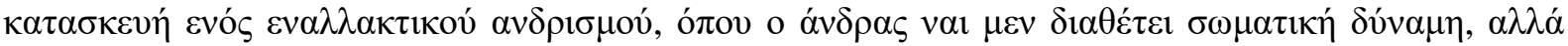

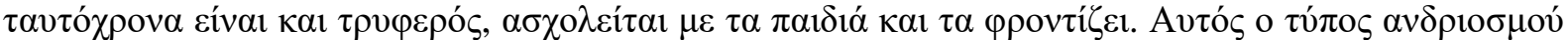

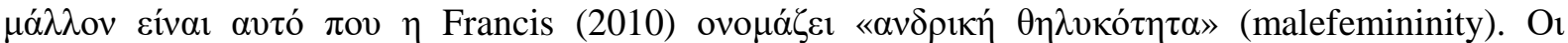

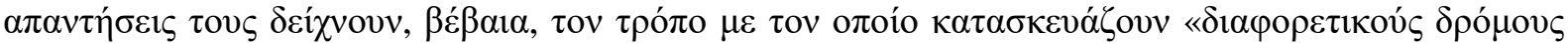

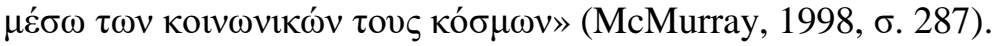

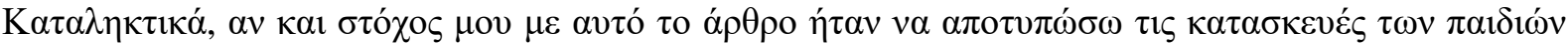

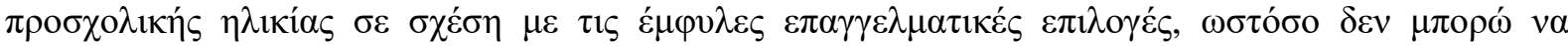

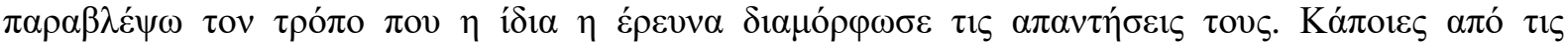

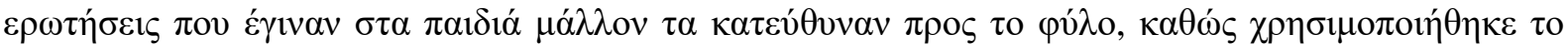

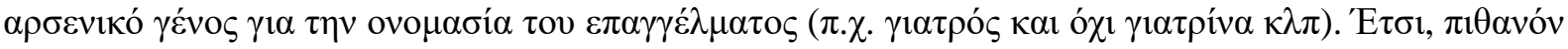

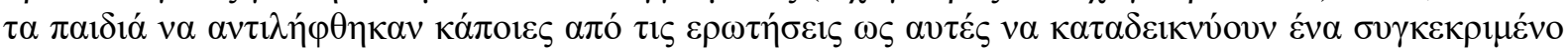

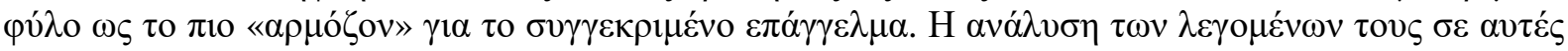

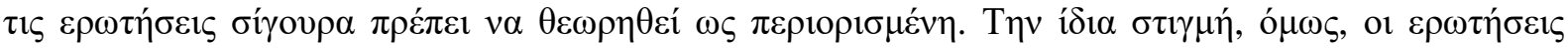

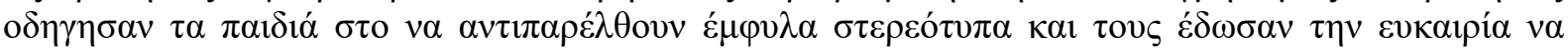

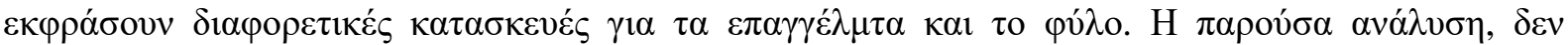
$\mu \pi$

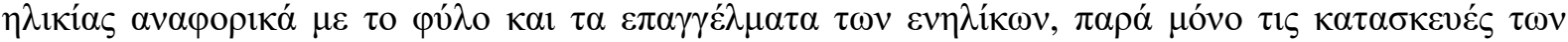

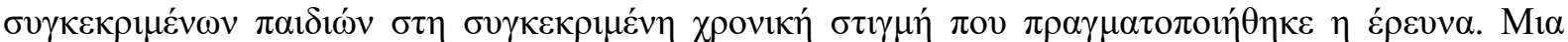

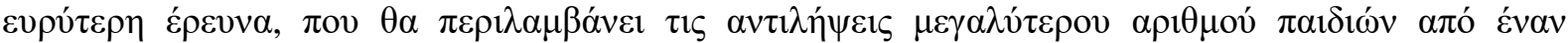

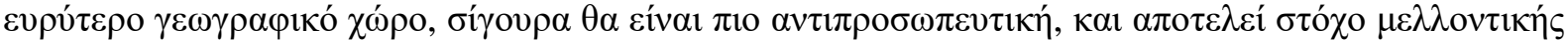

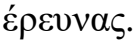

\section{ВIВАIОГРАФІА}

\section{$\Xi \varepsilon v o ́ \gamma \lambda \omega \sigma \sigma \eta$}

Adams, C. and Walkerdine, V. (1986) Investigating gender in the primary schools. Activity based inset materials for primary teachers. London: Inner London Educational Authority

Arber, S. and Ginn J. (1995) The mirage of gender equality: Occupational success in the labour market and within marriage. British journal of sociology, 46(1), pp. 21-43

Baker, C. and Davies, B. (1989) A lesson on sex roles. Gender and education, 1(1), pp. 59-75

Cameron, C. (2001) Promise or problem?A review of the literature on men working in early childhood services.Gender, work and organization, 8(4), pp. 430-453

Clarricoates, K. (1980) All in a day's work. In D. Spencer and E. Sarah (eds) Learning to lose: Sexism and education. London: The women's press, pp. 69-80

Cockburn, C. (1987) Two track training. London: Macmillan Education 
Cohen, L., Manion, L. and Morrison, K. (2013) Research methods in education. London: Routledge

Connell, R. (1995) Masculinities. Low Angeles: University of California Press

Davies, B. (1989) Frogs, snails and feminist tales. Sydney: Allan and Unwin

Delphy, C. and Leonard, D. (1992) Familiar exploitation. Cambridge: Polity Press

Drever, E. (1997) Using semi-structured interviews in small-scale research: A teacher's guide. Glasgow: Scottish Council of Research in Education

Epstein, D., Kehily, M., Mac anGhail, M. and Redman, P. (2001) Boys and girls come out to play. Men and masculinities, 4, pp. 158-172

Francis, B. (2010) Re/theorising gender: female masculinity and male femininity in the classroom?Gender and education, 22(5), pp. 477-490

Francis, B. J. (1996) Children's constructions of gender, power and adult occupation.Unpublished $P h D$ thesis. London: University of North London

Friedman, N. (2006) Nancy J. Hirschman on the social construction of women's freedom.Hypatia, 21(4), pp. 182-191

Goldstein, B. and Oldham, J. (1979) Children and work: A study of socialization. New York: Transaction Books

Hill, R. (1990) Involving men in the caring and educational services. Local government policy making, 17(3), pp. 36-39

Hsieh, H. F. And Shannon, S. E. (2005) Three approaches to qualitative content analysis. Qualitative health research, 15(9), pp. 1277-1288

Hutton, W. (1995) The state we're in. London: Vintage

Jensen, J. J. (1996) Men as workers in childcare services: A discussion paper. European commission network on childcare and other measures to reconcile employment and family responsibilities for women and men. Brussels: European equal opportunities unit

Kehily, M. (1999) More sugar? Teenage gender displays and sexual learning. European journal of cultural studies, 2(1), pp. 65-89

Kelly, A. (1989) "When I grow up I want to be...": A longitudinal study of the development of career preferences. British journal of guidance and counseling, 17(2), pp. 179-200

Levy, G. D., Sadovsky, A. L.And Troseth, G. L. (2000) Aspects of young children's perceptions of gendertyped occupations.Sex roles, 42 (11/12), pp. 993-1006

Lloyd, B. B. and Duveen, G. (1992) Gender identities and education: The impact of starting school. Hemel Hempstead: Harvest Wheatsheaf 
Lowe, K. (1998) Gendermaps. In N. Yelland (ed) Gender in early childhood. London: Routledge, pp. 206-222

McMurray, P. (1998) Gender behaviours in an early childhood classroom through an ethnographic lens.Qualitative studies in education, 11(2), pp. 271-290

Meehan, A. M. and Janik, L. M. (1990) Illusory correlation and the maintenance of sex role stereotypes in children.Sex roles, 22(1/2), pp. 83-95

Nemerowicz, G. (1979) Children's perceptions. New York: Praeger Publishers

Oliveira, I. M., Porfetli, E. J.And Do CeuTaveira, M. (2017) Children's career exploration and development: Reserach overview and agenda. In M. Watson and M. McMahon (eds) Career exploration and development in childhood Perspectives from theory, practice and research. London: Routledge, pp. 73-86

Osgood, J., Francis, B, \& Archer, L. (2006). Gendered identities and work placement: Why don't boys care? Journal of Education Policy, 21(3), pp. 305-321

Papanastasiou, E. (2016) Gender and leadership in Greek primary education.Unpublished PhD thesis. London: London Metropolitan University

Reay, D. (2001) 'Spice girls', 'nice girls' and 'tomboys': Gender discourses, girls vultures and femininities in the primary classroom. Gender and education, 13, pp. 153- 166

Robb, B. (1981) Maternal employment and children's sex role perceptions. Educational research, 23(3), pp. 223-225

Rosenthal, D. A. and Chapman, D. C. (1982) The lady spaceman: Children's perceptions of sex-role stereotyped occupations. Sex roles, 8, pp. 959-965

Sealey, A. and Knight, C. (1990) "We don't like talking in front of the boys": Talk and inequality. Education 3-13, 18, pp. 55-59

Sharpe, S. (1976) Just like a girl: How girls learn to be women. London: Penguin/Pelican

Spender, D. (1982) Invisible women: The schooling scandal. London: Writers and readers

Steedman, C, (1982) The tidy house: Little girls writing. London: Virago

Thorne, B. (1993) Gender play: Girls and boys in school. New Brunswick: Rutgers

Tremaine, L. (1982) Children's occupational sex-typing. Sex roles, 8(7), pp. 691-710

Tremaine, L. S. and Schau, C. J. (1979) Sex role aspects in the development of children's vocational knowledge.Journal of vocational behaviour, 14, pp. 317-328

Walkerdine, V. (1990) Schoolgirl fictions. London: Verso

Watson, M. and McMahon, M. (2005) Children's career development: A research review from a learning perspective.Journal of vocational behavior, 67(2), pp. 119-132 
Watson, M. and McMahon, M. (2017) Telling stories of childhood career development. In M. Watson and M. McMahon (eds) Career exploration and development in childhood Perspectives from theory, practice and research. London: Routledge, pp. 1-8

Whitehead, J. (1996) Sex stereotypes, gender identity and subject choice at A-level. Educational research, 38(2), pp. 147-160

Whyte, J. (1986) Girls into science and technology. London: Routledge and Kegan Paul

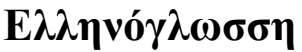

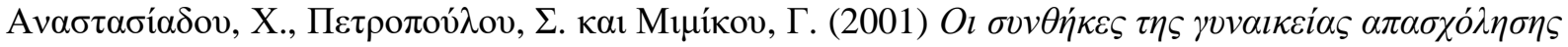

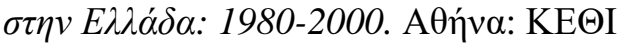

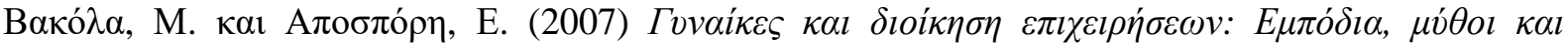

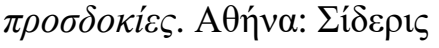

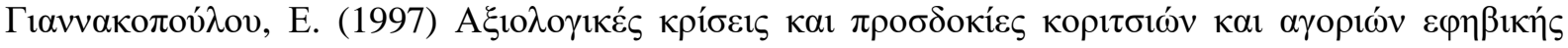

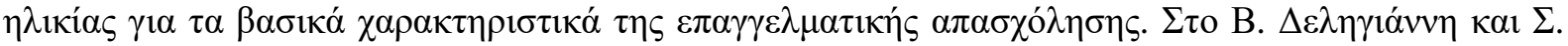

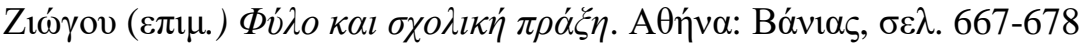

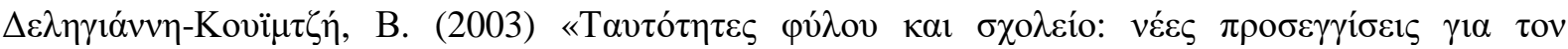

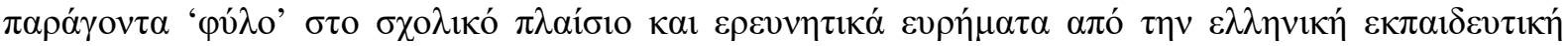

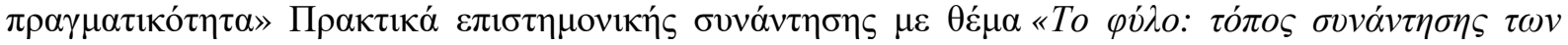

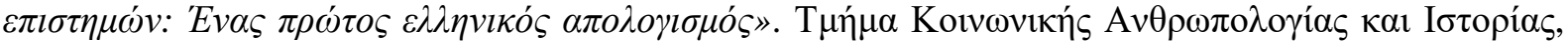

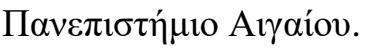

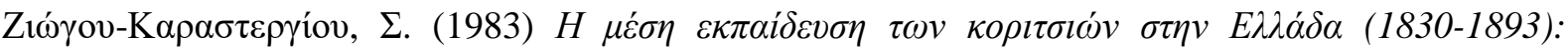

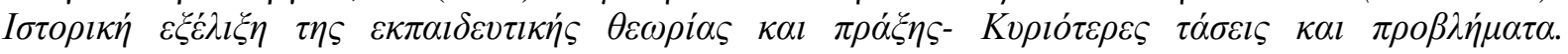

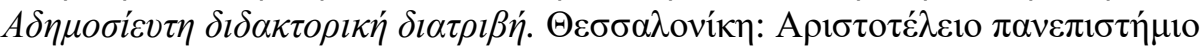

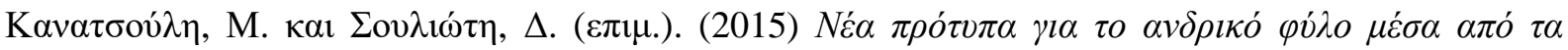

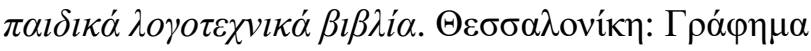

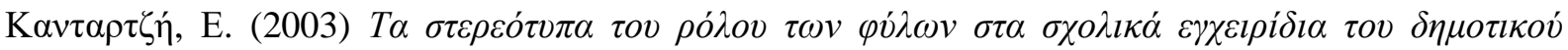

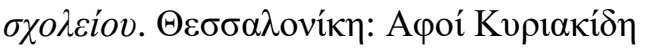

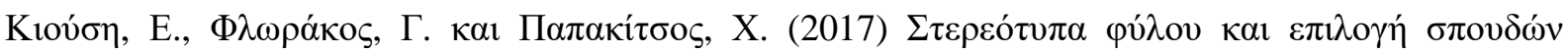

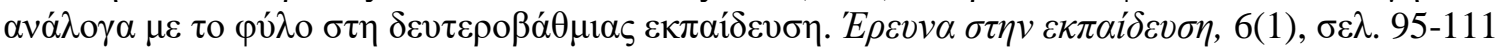

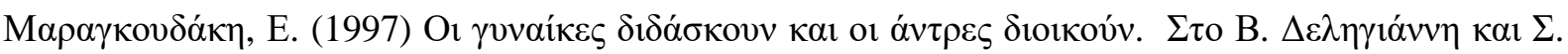

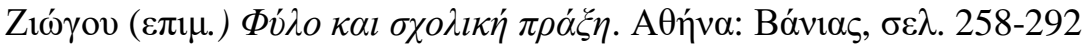

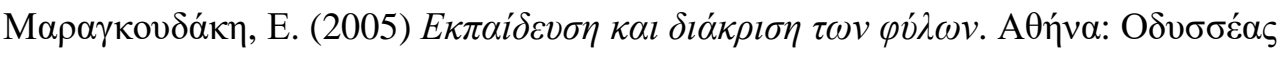

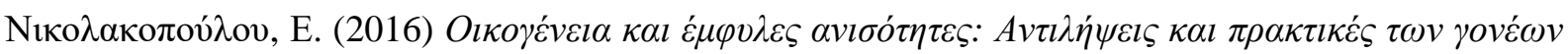

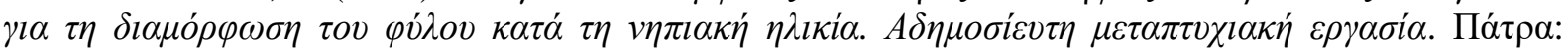
Avoıน

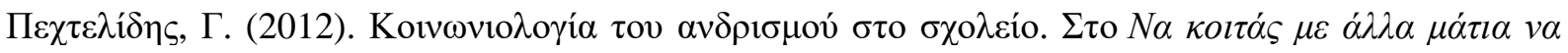

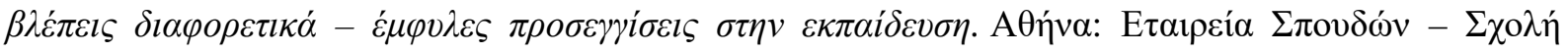

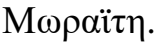




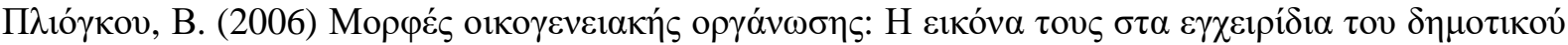

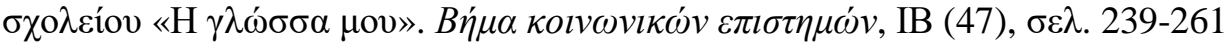

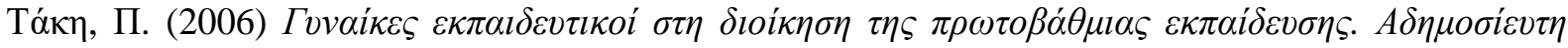

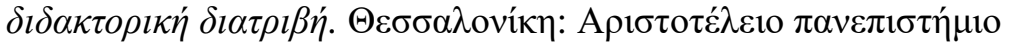

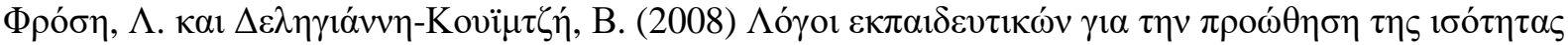

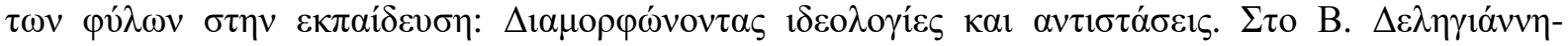

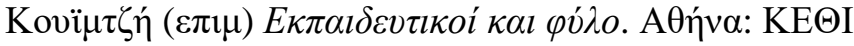

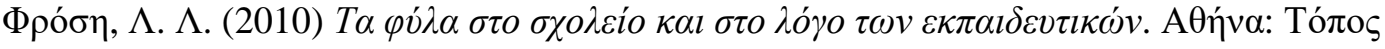

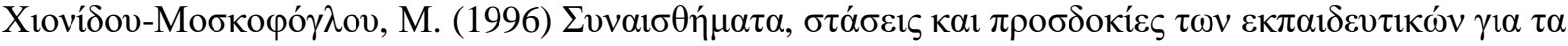

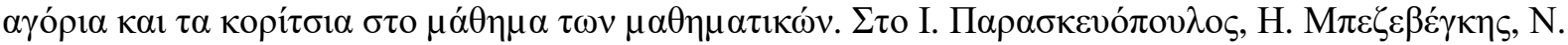

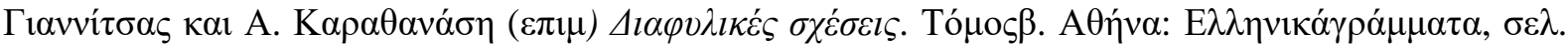
$302-314$

\section{$\Delta \mathrm{\iota \alpha \delta \iota \tau v \alpha \kappa \varepsilon ́s} \mathrm{\Pi \eta \gamma \varepsilon ́ \varsigma}$}

European Commission (2017) The gender pay gap in Greece. $\Sigma \tau$ o http://www.isotita.gr/wp-

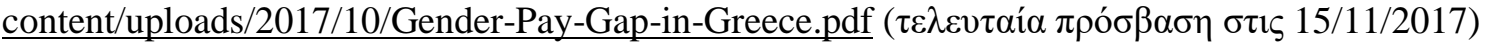

Eurostat (2017) Gender pay gap statistics. $\Sigma \tau$ o http://ec.europa.eu/eurostat/statisticsexplained/index.php/Gender_pay_gap_statistics ( $\tau \varepsilon \lambda \varepsilon v \tau \alpha \alpha i ́ \alpha \pi \rho o ́ \sigma \beta \alpha \sigma \eta ~ \sigma \tau \iota \varsigma$ 17/11/2017)

OECD (2017a) Employmentrate. $\Sigma \tau$ o https://data.oecd.org/emp/employment-rate.htm\#indicator-chart

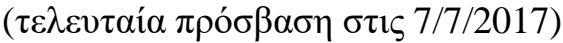

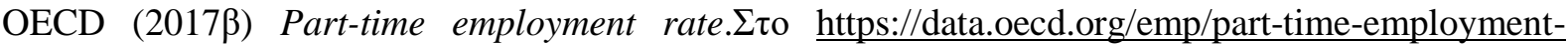
rate.htm ( $\tau \varepsilon \lambda \varepsilon v \tau \alpha i ́ \alpha \pi \rho o ́ \sigma \beta \alpha \sigma \eta ~ \sigma \tau ı \varsigma ~ 7 / 7 / 2017)$

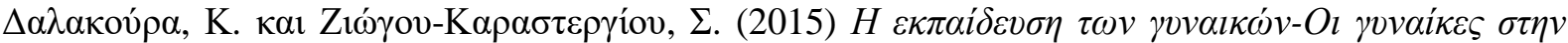

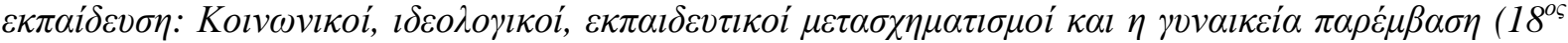

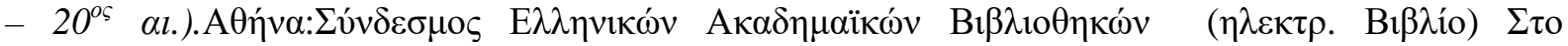
https://repository.kallipos.gr/pdfviewer/web/viewer.html?file=/bitstream/11419/2585/1/02_15210_Ma ster_Document.pdf ( $\left.\tau \varepsilon \lambda \varepsilon v \tau \alpha \alpha^{\prime} \alpha \pi \rho o ́ \sigma \beta \alpha \sigma \eta \sigma \tau 1 \varsigma 10 / 11 / 2017\right)$

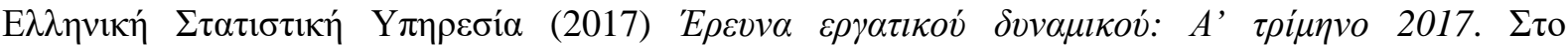
http://www.statistics.gr/el/statistics?p_p id=documents WAR publicationsportlet INSTANCE qDQ

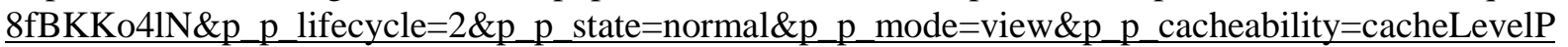
age \&p $\_$_ col id=column-

$2 \& p \quad p$ col count $=4 \& p$ p col pos $=1 \&$ documents WAR publicationsportlet INSTANCE qDQ8fB KKo41N_javax.faces.resource=document\&_documents_WAR_publicationsportlet_INSTANCE_qDQ 8fBKKo4lN_ln=downloadResources\&_documents_WAR_publicationsportlet_INSTANCE_qDQ8fB KKo4lN_documentID=252682\&_documents_WAR_publicationsportlet_INSTANCE_qDQ8fBKKo41 N_locale=el $(\tau \varepsilon \lambda \varepsilon v \tau \alpha i ́ \alpha \pi \rho o ́ \sigma \beta \alpha \sigma \eta 7 / 7 / 2017)$

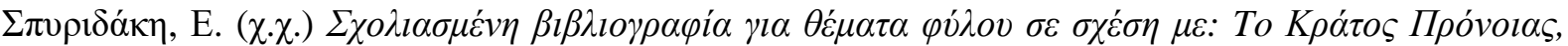

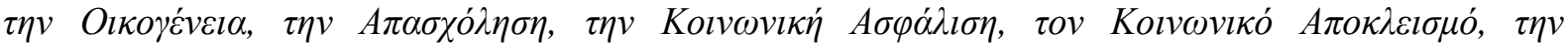

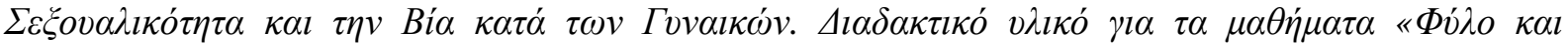

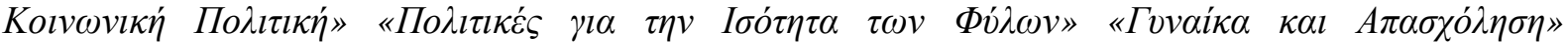

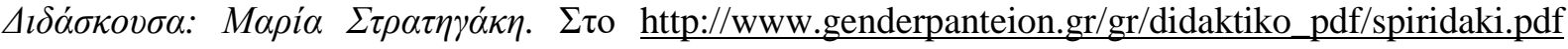

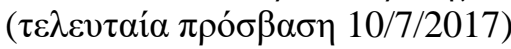




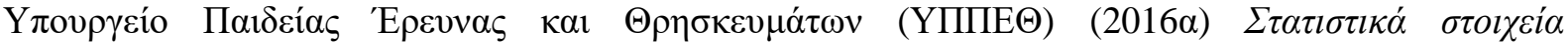
Пave $\lambda \alpha \delta$ iucóv $\varepsilon \xi \varepsilon \tau \dot{\alpha} \sigma \varepsilon \omega v$ : N'́o

$\Sigma \dot{v} \sigma \tau \mu \alpha$ 2016. https://www.minedu.gov.gr/publications/docs2016/\%CE\%A3\%CE\%A4\%CE\%91\%CE\%A4\%CE\%99 \%CE\%A3\%CE\%A4\%CE\%99\%CE\%9A\%CE\%91_\%CE\%9D\%CE\%95\%CE\%9F_\%CE\%A3\%CE\%

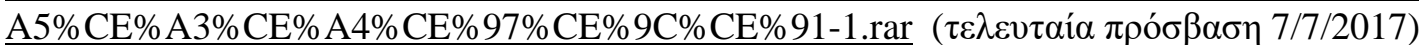

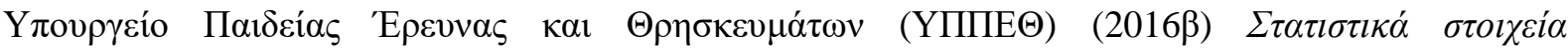
$\varepsilon \xi \xi \varepsilon \dot{\alpha} \sigma \varepsilon \omega v$ :

Падаı⿱宀 $\Sigma \dot{\sigma} \sigma \tau \mu \alpha$ 2016. https://www.minedu.gov.gr/publications/docs2016/\%CE\%A3\%CE\%A4\%CE\%91\%CE\%A4\%CE\%99 \%СЕ\%A3\%CE\%A4\%CE\% 99\%CE\%9A\%CE\%91 \%CE\%A0\%CE\%91\%CE\%9B\%CE\%91\%CE\%99 \%CE\%9F \%CE\%A3\%CE\%A5\%CE\%A3\%CE\%A4\%CE\%97\%CE\%9C\%CE\%91.rar ( $\pi \rho o ́ \sigma \beta \alpha \sigma \eta 7 / 7 / 2017)$ 